\title{
Análise da eficiência dos sorventes naturais lignocelulósicos na remediação de petróleo derramado em águas marinhas utilizando fibra de sisal
}

\author{
Analysis of the efficiency of natural lignocellulosic sorbents in the remediation of oil spilled in \\ marine waters using sisal fiber
}

Análisis de la eficacia de los sorbentes lignocelulósicos naturales en la remediación de hidrocarburos vertidos en aguas marinas utilizando fibra de sisal

\section{Resumo}

A produção do petróleo, aplicados em diversos setores da economia, pode desencadear impactos ambientais provenientes de derrames e vazamentos marinhos na exploração, transporte e armazenamento, capazes de destruir biomas. Para remediar áreas impactadas, são utilizadas barreiras de contenção e limpeza em ambiente marinho. As fibras lignocelulósicas apresentam uma alta capacidade de sorver o óleo, podendo facilmente servir como barreiras em um processo de derramamentos, evitando que a mancha de óleo se alastre, além de proporcionar a remediação do local impactado. A fibra de sisal é um material lignocelulósico e é encontrada em larga escala na natureza como subproduto de produções agrícolas além de serem materiais de fontes renováveis bem como biodegradáveis. O presente trabalho apresenta, como objetivo, realizar um estudo envolvendo testes realizados em laboratório a fim de verificar a eficiência da fibra de sisal in natura na sorção do petróleo da Bacia de Campos. As análises morfológicas da fibra, através do Microscópio Eletrônico de Varredura (MEV), permitiram o acesso a imagens ampliadas da área superficial da fibra, compostas por poros responsáveis pelo processo de adsorção do óleo. Para avaliar a sua eficiência, ensaios de derramamentos offshore foram simulados, sob períodos distintos (5, 20, 40, 60, 90 e 120min), utilizando a mesa reciprocante $(126 \mathrm{rpm})$, que simula a hidrodinâmica marinha. Os dados colhidos sugeriram a necessidade de um tempo mínimo de 5 minutos de contato com óleo em eventuais derramamentos marinhos. A reciclagem da fibra foi analisada ao longo de seis ciclos subsequentes. O potencial adsortivo do último ciclo decaiu aproximadamente $12 \%$ da primeira sorção, caracterizando uma boa reutilização da fibra após sucessivas aplicabilidades.

Palavras-chave: Adsorção; Fibra de sisal; Derramamento de petróleo.

\begin{abstract}
Oil production, applied in various sectors of the economy, can trigger environmental impacts from marine spills and spills in exploration, transportation and storage, capable of destroying biomes. To remedy impacted areas, containment and cleaning barriers in a marine environment are used. Lignocellulosic fibers have a high capacity to absorb oil, and can easily serve as barriers in a spill process, preventing the oil stain from spreading, in addition to providing remediation of the impacted site. Sisal fiber is a lignocellulosic material and is found on a large scale in nature as a by-product of agricultural production in addition to being materials from renewable sources as well as biodegradable. The present work aims to carry out a study involving tests carried out in the laboratory in order to verify the efficiency of fresh sisal fiber in the sorption of oil in the Campos Basin. The morphological analysis of the fiber, through the Scanning Electron Microscope (SEM), allowed access to enlarged images of the surface area of the
\end{abstract}


fiber, composed of pores responsible for the oil adsorption process. To assess its efficiency, offshore spill tests were simulated, under different periods $(5,20,40,60,90$ and 120 minutes), using the reciprocating table (126 rpm), which simulates marine hydrodynamics. The data collected suggested the need for a minimum time of 5 minutes of contact with oil in eventual marine spills. Fiber recycling was analyzed over six subsequent cycles. The adsorptive potential of the last cycle dropped approximately $12 \%$ from the first sorption, characterizing good fiber reuse after successive applications.

Keywords: Adsorption; Sisal fiber; Oil spill.

\section{Resumen}

La producción de petróleo, aplicada en varios sectores de la economía, puede desencadenar impactos ambientales por derrames marinos y derrames en exploración, transporte y almacenamiento, capaces de destruir biomas. Para remediar las áreas impactadas, se utilizan barreras de contención y limpieza en un ambiente marino. Las fibras lignocelulósicas tienen una alta capacidad para absorber aceite y pueden servir fácilmente como barreras en un proceso de derrame, evitando que la mancha de aceite se propague, además de proporcionar remediación del sitio impactado. La fibra de sisal es un material lignocelulósico y se encuentra a gran escala en la naturaleza como subproducto de la producción agrícola, además de ser materiales de fuentes renovables y biodegradables. El presente trabajo tiene como objetivo realizar un estudio de ensayos realizados en laboratorio para verificar la eficiencia de la fibra fresca de sisal en la sorción de aceite en la Cuenca de Campos. El análisis morfológico de la fibra, a través del Microscopio Electrónico de Barrido (SEM), permitió acceder a imágenes ampliadas de la superficie de la fibra, compuesta por poros responsables del proceso de adsorción del aceite. Para evaluar su eficiencia, se simularon pruebas de derrames costa afuera, en diferentes períodos $(5,20,40,60,90$ y 120 minutos), utilizando la mesa recíproca (126 rpm), que simula la hidrodinámica marina. Los datos recopilados sugirieron la necesidad de un tiempo mínimo de 5 minutos de contacto con el petróleo en eventuales derrames marinos. El reciclaje de fibra se analizó durante seis ciclos posteriores. El potencial adsortivo del último ciclo disminuyó aproximadamente un 12\% desde la primera sorción, caracterizando una buena reutilización de la fibra luego de sucesivas aplicaciones.

Palabras clave: Adsorción; Fibra de sisal; Derrame de petróleo.

\section{Introdução}

O cenário do petróleo brasileiro sofreu mudanças significativas na última década. A produção média do petróleo saiu de 41 mil barris de petróleo por dia, para 2,94 milhões, registrado até o ano 2020. Esse aumento deve-se a lei 13375, sancionada em 2016, que declara o regime de partilha da produção do petróleo na camada Pré-Sal, localizada na bacia de Campos e de Santos. Atualmente, empresas brasileiras e estrangeiras podem produzir nesse local (Petrobrás, 2018; ANP, 2021).

Segundo dados retirados da ITOPF (2020), o número de grandes derrames de petróleo sofreu uma queda acentuada desde o relato dos primeiros incidentes na década de 1970. Nesta época, foram relatadas, em média, 24,5 acidentes/ano, reduzidos para 1,8, registrados até 2020. Esta diminuição é justificada pela pressão exercida pelos órgãos ambientais sob os setores das indústrias petroleiras a investirem mais em métodos de prevenção aos acidentes (Lopes, 2007; EPA, 2021).

Apesar desta redução considerável, os acidentes ainda persistem, sendo responsáveis por diversas devastações ambientais. Recentemente, em maio de 2019, foi relatado um grande acidente no América do Norte que liberou cerca 700 toneladas de petróleo para o meio ambiente e no Brasil ocorreu um acidente em foram liberadas mais de 5 mil toneladas de petróleo venezuelano, gerando impactos consideráveis para os locais atingindo, inclusive, a região costeira (ITOPF, 2020; Oliveira, 2020).

O petróleo é formado por compostos aromáticos diversos, além dos hidrocarbonetos saturados e dos componentes orgânicos nitrogenados sulfonados e oxigenados. Quanto maior o número de cadeias de anéis aromáticos, mais tóxico o petróleo será. Os principais poluentes aromáticos são os compostos Benzeno, Tolueno e Xileno (BTX), bem como os Hidrocarbonetos Policíclicos Aromáticos (HPAs), substâncias consideradas precursoras de ações mutagênicas e tumorais em sistemas biológicos (Trigo et al., 2007; Queiroz, 2011; Podgorski, et al., 2012; Speight \& El-Gendy, 2017).

Após um derramamento, o impacto gerado é de difícil recuperação, tornando necessário aplicar técnicas remediadoras para limpar o ambiente afetado. Atualmente são aplicadas técnicas diversas utilizando processos mecânicos, físicos, químicos 
ou biológicos para degradar e o óleo de forma rápida. Entretanto, o uso de muitas dessas técnicas pode gerar problemas ainda maiores para o meio ambiente, como, por exemplo, a emissão de gases poluentes liberados pela queima in situ e aumento do risco a vida marinha causado pela toxicidade dos dispersantes químicos (Cardoso et al., 2016; García-Garrido et al., 2016; Li et al., 2016; CETESB, 2021; Grotea et al., 2018).

Outra tecnologia aplicada atualmente com o objetivo de remediar ambientes impactados por petróleo, são os adsorventes (CETESB, 2021). Eles apresentam características favoráveis ao processo de adsorção pois são oleofílos, hidrofóbicos, de fácil recuperação após a sorção, além de apresentarem alta resistência a deformação e uma boa eficiência no seu uso reciclável (Lim \& Huang, 2007; Abdullah et al., 2010; Li et al., 2016). Existe três tipos: minerais, sintéticos e naturais. Os adsorventes sintéticos são os principais utilizados por possuírem uma alta capacidade de sorver o óleo. Entretanto, além de não possuírem fonte renovável, possuem baixo potencial de degradação e um alto custo em comparação aos outros tipos de adsorventes (Wang et al., 2012; Dong, 2015; CETESB, 2021).

A utilização dos adsorventes naturais está atribuída a fatores ambientais e econômicos uma vez que as fibras estão dispostas na natureza, possuindo fonte renovável, além de serem biodegradáveis possuindo um fácil descarte. A maioria dos adsorventes naturais são resíduos de produtos agrícolas e a sua utilização gera uma redução da poluição além de reduzir também os gastos com a taxa de descarte gerando, assim, uma maior economia (Lim \& Huang et al., 2007; Abdullah et al., 2010; CETESB, 2021; Wong, 2016).

Os adsorventes naturais possuem a capacidade de reter o petróleo principalmente a partir da transferência de massa denominada adsorção. Este fenômeno ocorre através da migração das espécies conhecidas como adsorvado, para a superfície do sólido poroso que equivale ao adsorvente. A força motriz é a diferença de concentração entre essas espécies que podem ser adsorvidas através de um processo químico (quimiossorção) ou físico (fisiossorção) (Lim \& Huang, 2007; Berger \& Bhown, 2011; Wang \& Guo, 2020).

A fibra de sisal (Agave sisalana) é um material que apresenta alta produção no Nordeste brasileiro, devido ao clima favorável ao plantio. Trata-se de uma fibra lignocelulósica com composição química marjoritárias de lignina, hemicelulose e celulose, além de outras frações de compostos como ceras e extrativos. O interesse no uso desta fibra na remediação de ambientes impactados por petróleo, pode ser atribuido a sua fonte renovável, a sua biodegradação e por apresentar caracteristicas hidrofóbicas bem como oleofílicas. Este material apresenta, ainda, uma porosidade responsável pela adsorção do petróleo (Annunciado et. al., 2005; Martin et. al., 2009; Barbosa, 2011; Segundo, 2011).

\section{Metodologia}

\section{Materiais e Métodos}

\section{Materiais}

$\mathrm{O}$ adsorvente utilizado no presente trabalho foi a fibra lignocelulósico de sisal, preservando suas características inatas. Este material foi obtido na empresa de Abaeté Gesso, localizada na cidade de Lauro de Freitas, na Bahia. Não foram realizados tratamentos que mudassem a estrutura da fibra, objetivando diminuir custos operacionais em possíveis aplicações em escala real.

Para simulações do derramamento de óleo, o petróleo da Bacia de Campos (Macabu-Coquinos) foi utilizado em todos os testes da eficiência de sorção pela fibra de sisal. Todos os testes foram realizados no Laboratório de Estudos do Petróleo (LEPETRO) do Núcleo de Estudos Ambientais (NEA), localizada no Instituto de Geociência (IGEO) da Universidade Federal da Bahia (UFBA) e o petróleo foi doado pela Petrobrás. 


\section{Métodos}

- $\quad$ Caracterização do petróleo

Para realizar a caracterização do petróleo e avaliar o seu grau de fluidez, um medidor de densidade (VIDA 40) foi utilizado. A temperatura referente ao estudo foi de $20^{\circ} \mathrm{C}$, visto que todos os testes com este óleo foram realizados nesta faixa. A partir deste resultado, a densidade relativa do fluido $\left({ }^{\circ} \mathrm{API}\right)$ pôde ser determinada e posteriormente classificada.

\section{- $\quad$ Análise morfológica das fibras}

A estrutura morfológica da fibra foi analisada utilizando o Microscópio Eletrônico de Varredura (MEV) no Laboratório LAMUME, localizado no Instituto de Física da UFBA. O objetivo da análise é obter, em imagens ampliadas, a porosidade da fibra responsável pela captação do óleo adsorvido. A imagem foi ampliada em 350x, para permitir a melhor visualização do adsorvente.

\section{- Amostra}

Para confeccionar as amostras, foram ensacadas 0,5 gramas da fibra de sisal em um material polimérico padronizados em tamanhos de 2x8 cm, visando facilitar a coleta da amostra após os testes de sorção do petróleo. Este material contém pequenos furos para permitir a migração do óleo para a fibra. As amostras foram inseridas em pequenos potes de vidro para permitir a sua pesagem.

- $\quad$ Capacidade de sorção do óleo

\section{Teste Cinético}

Para obter o tempo de maior eficiência, as amostras foram inseridas em Erlenmeyers contendo $200 \mathrm{~mL}$ de água marinha artificial e $5 \mathrm{~mL}$ do petróleo da Bacia de Campos. Todos os ensaios foram realizados na temperatura de $20^{\circ} \mathrm{C}$. Para simular a hidrodinâmica marinha, os Erlenmeyers foram inseridos em uma mesa agitadora (MARCONI), com rotação de 126rpm. Os tempos de permanência de contato óleo-fibra estudados foram de 5, 20, 40, 60, 90 e 120 min.

Segundo (2011), relatou em seu trabalho que a fibra de sisal contém características hidrofílicas e, desta maneira, também pode adsorver a água. A partir disso, as amostras, após o teste de sorção nos intervalos predeterminados, foram congeladas e posteriormente inserida no liofilizador (L108), com o objetivo de obter as amostras desidratadas, somente com a massa de óleo adsorvida.

Para a estimativa do teor de óleo adsorvido pela fibra de sisal, as equações 1 e 2 foram utilizadas. Como o objetivo da presente pesquisa é estudar o potencial adsortivo pela fibra, foram realizados testes somente com o material polimérico e a massa de óleo obtida por ele foi subtraída da massa de óleo da amostra após a sorção, proporcionando um resultado próximo do valor real de óleo adsorvido pelo sisal. Os testes, para todos os períodos pré-determinados, foram realizados em triplicada obtendo uma relação da massa de óleo, em gramas, capaz de ser adsorvida por cada grama de fibra (Annunciado et. al., 2005; Al Zubaidy et al., 2015; Ferreira, 2009).

$$
\begin{aligned}
& M_{\text {Polietileno }}=M_{P E f}-M_{P E i} \\
& S_{f i b r a}=\frac{M_{f}-M_{i}-M_{\text {Polietileno }}}{M_{\text {fibra }}}
\end{aligned}
$$

Sendo,

$$
M_{\text {polietileno }}=\text { Massa de óleo adsovida pelo polietileno }(g)
$$




$$
\begin{aligned}
& M_{P E i}=\text { Média da massa inicial do polietleno }(g) \\
& M_{P E f}=\text { Média da massa final do polietileno desidratada }(g) \\
& S_{f i b r a}=\text { Sorção do óleo pela fibra }\left(g_{b i t e o} / g_{f i b r a}\right) \\
& M_{i}=\text { Média da massa inicial da amostra }(g) \\
& M_{f}=\text { Média da massa final da amostra desidratada }(g) \\
& M i_{f i b r a}=\text { Média da massa inicial da fibra }(g)
\end{aligned}
$$

\section{Reciclagem da fibra}

$\mathrm{O}$ teste de reciclagem da fibra foi realizado de forma semelhante ao teste anterior. As amostras que obtiveram uma maior eficiência correspondente ao melhor período de contato do óleo com a fibra, foi inserida em uma centrífuga (CENTRI 310), com rotação de $2500 \mathrm{rpm}$, no tempo de 30 minutos. Esta técnica foi utilizada visando a dessorção do óleo, através do processo de separação do óleo presente na fibra. Em seguida, as amostras centrifugadas foram pesadas, compondo a nova massa inicial do segundo ciclo (primeira reciclagem). Um novo teste de sorção foi realizado com as mesmas quantidades de água e óleo utilizadas anteriormente no período de maior eficiência, obtendo as novas massas de óleo adsorvidas para este ciclo. Ao todo, foram realizados seis ciclos totalizando cinco reutilizações a fim de estudar o potencial de reutilização da fibra de sisal.

\section{Resultados e Discussão}

\section{Análise morfológica da fibra}

A interface da fibra de sisal está demonstrada na Figura 1, obtida através do Microscópio Eletrônico de Varredura

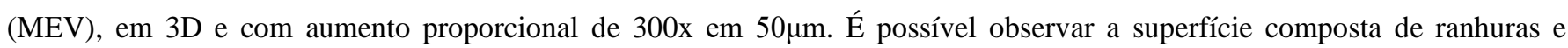
porosidade correspondente da fibra na face lateral e frontal, favorecendo a captação do petróleo através da infiltração do petróleo por estes canais existentes na extensão do material adsorvente (Annunciado et. al., 2005; Lim \& Huang, 2007; Wang \& Guo, 2020; Abdullah et al., 2010; Li et al., 2016).

Figura 1. Análise morfológica da fibra de sisal.

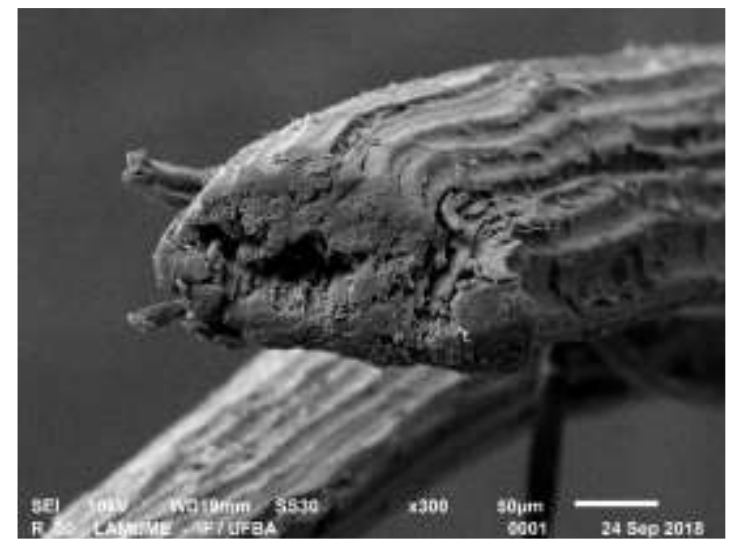

Fonte: Autores. 


\section{Caracterização do óleo}

A Tabela 1 descreve os dados coletados quanto a ${ }^{\circ}$ API do petróleo da Bacia de Campos, na temperatura de $20^{\circ} \mathrm{C}$. Este estudo é realizado a fim de coletar informação do grau de fluidez o óleo que, segundo a classificação da OPEP (2018), é classificado como pesado, estando em uma faixa superior a 10,5 e inferior a 26. Este resultado sugere que, na temperatura de $20^{\circ} \mathrm{C}$, a fluidez do óleo permite um contato constante com a fibra (Barragan, 2012; Sousa, 2009).

Tabela 1. Propriedades da amostra do petróleo da Bacia de Campos (Macabu-Coquinos).

\begin{tabular}{ccc}
\hline $\mathrm{T}\left({ }^{\circ} \mathrm{C}\right)$ & Densidade $\left(\mathrm{g.cm}^{-3}\right)$ & ${ }^{\circ}$ API \\
\hline 20 & $\mathbf{0 , 9 0 4}$ & $\mathbf{2 4 , 9 0}$ \\
\hline
\end{tabular}

Fonte: Autores.

\section{Capacidade de sorção do óleo}

- Obtenção do tempo de maior eficiência

O Figura 2 (a) exibe o potencial adsortivo pela fibra de sisal nos intervalos definidos. É possível observar que no tempo de 60 minutos, a eficiência foi superior aos demais, com um valor de sorção próximo de 4,3 gramas de óleo para cada grama de fibra $( \pm 0,003)$, adsorvendo cerca de $50 \%$ do óleo inserido no inicio do teste, conforme mostrado na Figura 2 (b). A diferença inferior a 5 e 3\% nos testes em 20 e 90 minutos, respectivamente, em relação ao tempo de maior sorção, demostram a equivalências em seus resultados. A boa eficiência na sorção no tempo de 5 minutos (3,235 $\pm 0,005 \mathrm{~g}$ óleo/g fibra), com uma adsorção próxima de $36 \%$ da quantidade de óleo utilizada inicialmente, sugere a necessidade de um tempo mínimo de contato do óleo com a fibra, como é possível observar nos valores de massa de óleo exibidas na Tabela 2. No tempo de 40 minutos, que exibiu menor eficiência, obteve uma sorção significativa próxima de 32\% (2,876 $\pm 0,002 \mathrm{~g}$ óleo/g fibra). 
Figura 2. Potencial adsortivo pela fibra de sisal nos intervalos definidos. (a) Sorção de óleo para cada/ grama de fibra (b) Porcentagem de óleo adsorvida.

(a)

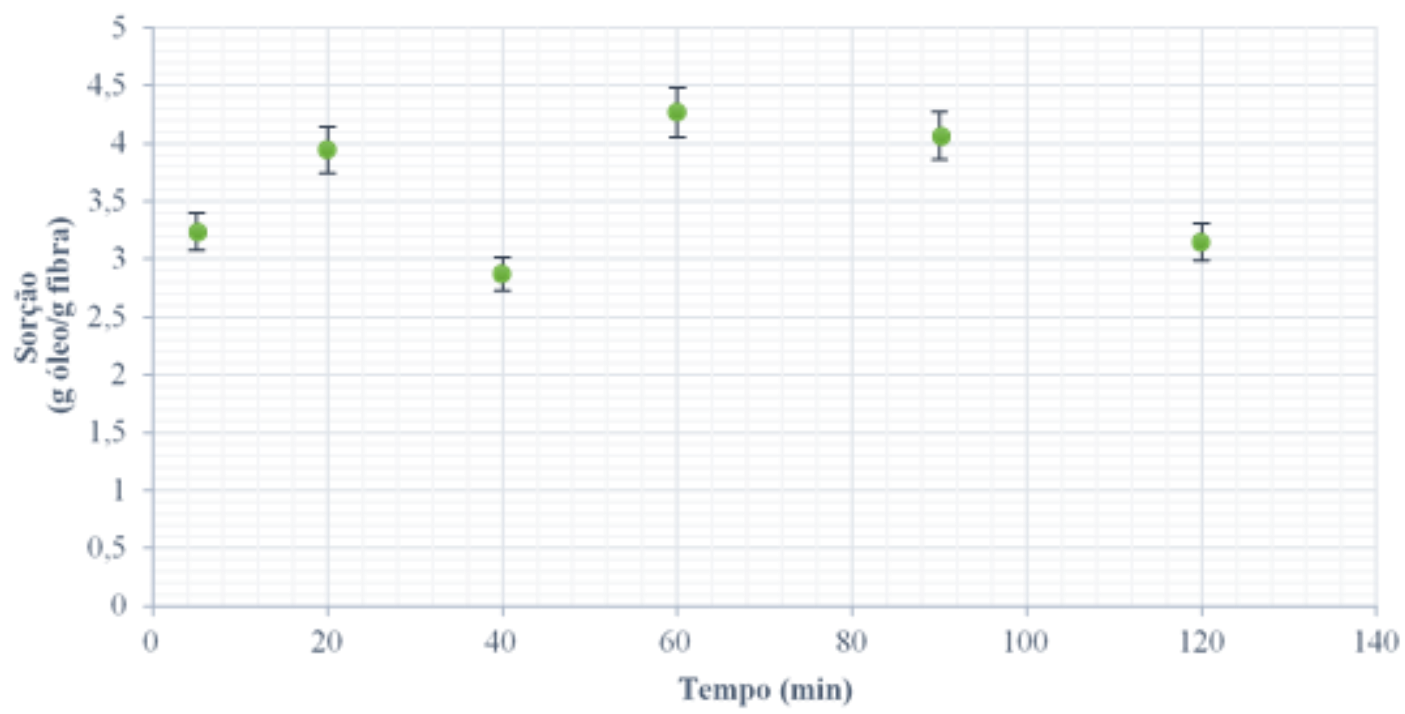

(b)

60

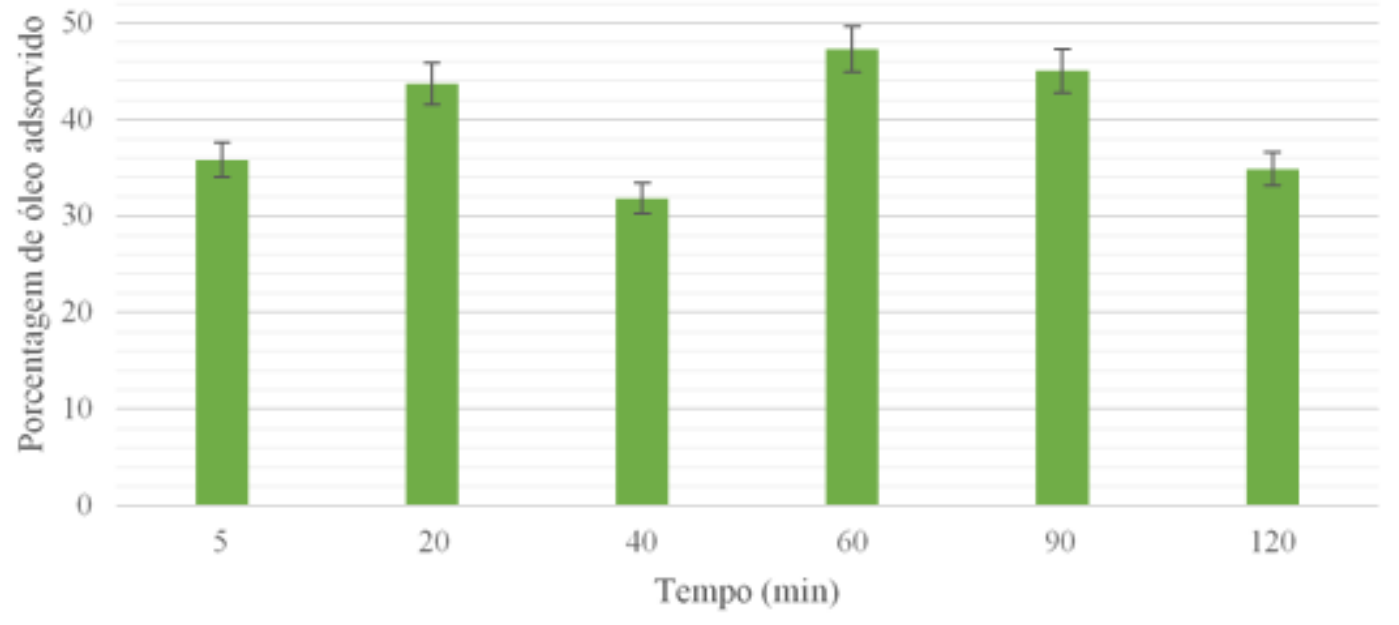

Fonte: Autores.

Tabela 2. Valores da sorção e da massa de óleo da Bacia de Campos pela fibra de sisal desidratada nos intervalos definidos.

\begin{tabular}{c|cccc}
\hline \multirow{2}{*}{ Tempo (min) } & Massa de óleo $(\mathbf{g})$ & $\begin{array}{c}\text { Sorção } \\
\text { (g óleo/g fibra) }\end{array}$ & $\begin{array}{c}\text { Desvio } \\
\text { Padrão }\end{array}$ & Porcentagem de óleo adsorvida \\
\hline $\mathbf{5}$ & 1,619 & 3,235 & $\pm 0,005$ & 35,83 \\
\hline $\mathbf{2 0}$ & 1,975 & 3,944 & $\pm 0,004$ & 43,69 \\
\hline $\mathbf{4 0}$ & 0,784 & 2,876 & $\pm 0,002$ & 31,82 \\
\hline $\mathbf{6 0}$ & 2,165 & 4,269 & $\pm 0,002$ & 47,91 \\
\hline $\mathbf{9 0}$ & 2,035 & 4,065 & $\pm 0,003$ & 45,02 \\
\hline $\mathbf{1 2 0}$ & 1,576 & 3,148 & $\pm 0,005$ & 34,87 \\
\hline
\end{tabular}

Fonte: Autores. 
Os valores de sorção com o passar do tempo não indicam a proporcionalidade visto que a hidrodinâmica marinha, simulada pela mesa, possibilita a dessorção da fibra. Estes resultados sugerem a importância que exerce os estudos dos fatores meteorológicos como, por exemplo, a influência da velocidade do vento, alterando as condições marinhas, que possibilita mudanças positivas ou negativas para a aplicabilidade da técnica. Para diferentes rotações, o tempo de maior eficiência pode sofrer modificações.

A Figura 3 apresenta uma análise comparativa da sorção do óleo antes e após a desidratação. A analogia é realizada em virtude do caráter hidrofílico da fibra de sisal estudado. Os resultados indicam uma acentuada discrepância relativa ao valor real da sorção do óleo (desidratada), evidenciando a influência da água nos experimentos (Tabela 3). Como esperado, a fibra obteve valores superiores na etapa em que antecede a desidratação (pós sorção), para todos os tempos estudados na presente pesquisa. Em 60min, a discrepância da sorção de óleo foi cerca de 20\%. A influência da água tem maior percepção no teste de 40min. A discrepância referente a este tempo é superior a 90\%, com uma sorção de quase 2 vezes o peso do óleo real adsorvido medido após a desidratação. Estipula-se que as moléculas de água ocuparam os sítios disponíveis interferindo no processo de adsorção do óleo. Este comportamento caracteriza uma justificativa hipotética para os menores resultados de sorção do óleo pertinente a este tempo.

Figura 3. Comparação da capacidade de sorção da água e do óleo pela fibra de sisal.

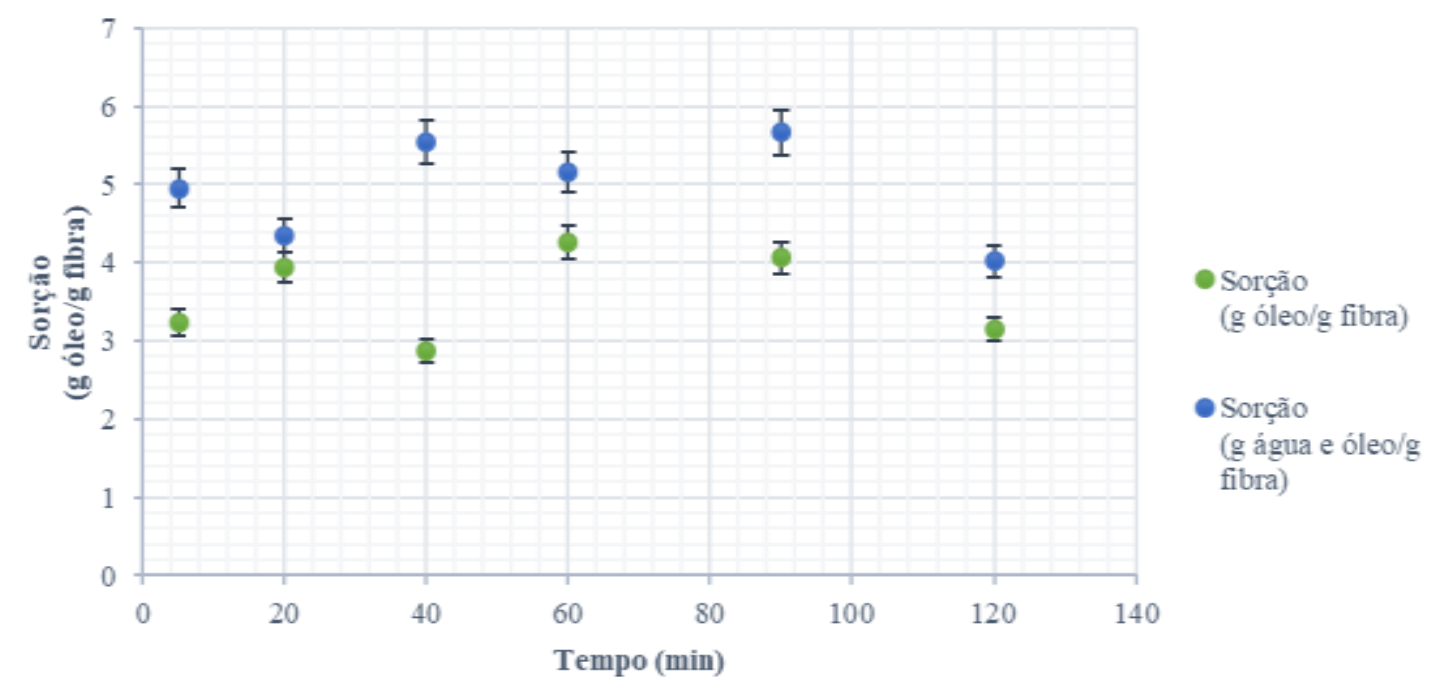

Fonte: Autores.

Tabela 3. Analise da influência da sorção da água pela fibra de sisal.

\begin{tabular}{c|ccc}
\hline Tempo (min) & $\begin{array}{c}\text { Sorção } \\
\text { (g óleo/g fibra) } \\
\text { desidratada }\end{array}$ & $\begin{array}{c}\text { Sorção } \\
\text { (g água e óleo/g fibra) }\end{array}$ & $\begin{array}{c}\text { Discrepância } \\
\text { relativa }(\%)\end{array}$ \\
\hline $\mathbf{5}$ & 3,235 & 4,954 & 53,13 \\
\hline $\mathbf{2 0}$ & 3,944 & 4,348 & 10,24 \\
\hline $\mathbf{4 0}$ & 2,876 & 5,538 & 92,60 \\
\hline $\mathbf{6 0}$ & 4,269 & 5,163 & 20,95 \\
\hline $\mathbf{9 0}$ & 4,065 & 5,663 & 39,30 \\
\hline $\mathbf{1 2 0}$ & 3,148 & 4,017 & 27,57 \\
\hline
\end{tabular}

Fonte: Autores. 
$\mathrm{g} / \mathrm{cm}^{3}$ à $\left.20^{\circ} \mathrm{C}\right)$ por diferentes fibras em tempos pré-estabelecidos (5, 20, 40, 60 e 1440 minutos). Em um dos testes narrado pelo autor, o potencial adsortivo da fibra de sisal in natura é estudada. Os resultados alcançados pelo autor referem-se a $10 \mathrm{~mL}$ de petróleo para cada teste realizado. Sob condições estáticas, o percentual de óleo adsorvido sob o tempo de 60min foi superior a 60\% (6,4 g óleo/g fibra), não havendo alta variação para o ensaio em sistema dinâmico. Entretanto, a técnica aplicada para quantificação de óleo, descrita, não subtrai a massa de água presente na amostra após o teste.

Estudos realizados por Segundo (2011), evidenciam a sorção do biodiesel $\left(0,97 \mathrm{~g} / \mathrm{cm}^{3}\right.$ à $\left.25^{\circ} \mathrm{C}\right)$ pela fibra de sisal sob os tempos de 5 10, 20, 60 e 1440 minutos. A amostra $(0,5 \mathrm{~g})$, isentas de tratamentos, foi exposta a $50 \mathrm{~mL}$ de óleo. O sisal apresentou maior eficiência adsortiva no intervalo de $60 \mathrm{~min}$, obtendo peso de 1,24 g, ao final do teste. Desta maneira, a fibra aderiu $0,74 \mathrm{~g}$ de óleo adicionado. A metodologia nos testes narrado pelo autor, no entanto, são isentos de água e sob condições estáticas, não sendo simuladas situações equiparáveis com derramamentos reais.

- Reciclagem da fibra

O critério utilizado para avaliar a eficiência da reutilização da fibra de sisal correspondeu ao número de ciclos de sorções em que o sorvente foi submetido sem que houvesse um alto desgaste que limitasse a captação do óleo após o uso da técnica, devido a diminuição do potencial de sorção de óleo para cada grama de fibra. Desta maneira, foram avaliados os seis ciclos (seis sorções) que compuseram a análise.

A reutilização da fibra de sisal após o sexto ciclo de sorção é mostrado na Figura 4. O potencial adsortivo da fibra na primeira sorção era de $4,330 \mathrm{~g}$ de óleo/g fibra $( \pm 0,002)$, reduzindo em cerca de $5 \%$ após o terceiro ciclo $(4,141 \pm 0,002 \mathrm{~g}$ de óleo/g fibra), conforme mostrado na Tabela 6. O comportamento decrescente no processo de reciclagem da fibra também foi relatado por Wang et al. (2012), que aborda, em seus estudos, o potencial uso da fibra Kapok para adsorver tolueno. O processo de dessorção, narrado pelo autor, utiliza um pistão para retirada do óleo. Os resultados da reutilização também foram semelhantes ao trabalho de Abdullah et al. (2010), que estuda a adsorção do óleo diesel pela fibra de Sumaúma. A técnica para remoção de óleo descrita pelo autor, é análoga ao da presente pesquisa, fazendo uso de uma centrifuga na rotação de 3000 rpm, durante 30 min. Tanto Wang et al. (2012) quanto Abdullah et al. (2010) descrevem um declínio de sorção de 30\% até o terceiro ciclo. 
Figura 4. Reciclagem da fibra de sisal avaliada sob seis ciclos subsequentes de sorção do óleo da Bacia de Campos. (a) Sorção de óleo para cada/ grama de fibra (b) Porcentagem de óleo adsorvido em relação ao primeiro teste de sorção (reutilização 0).

(a)

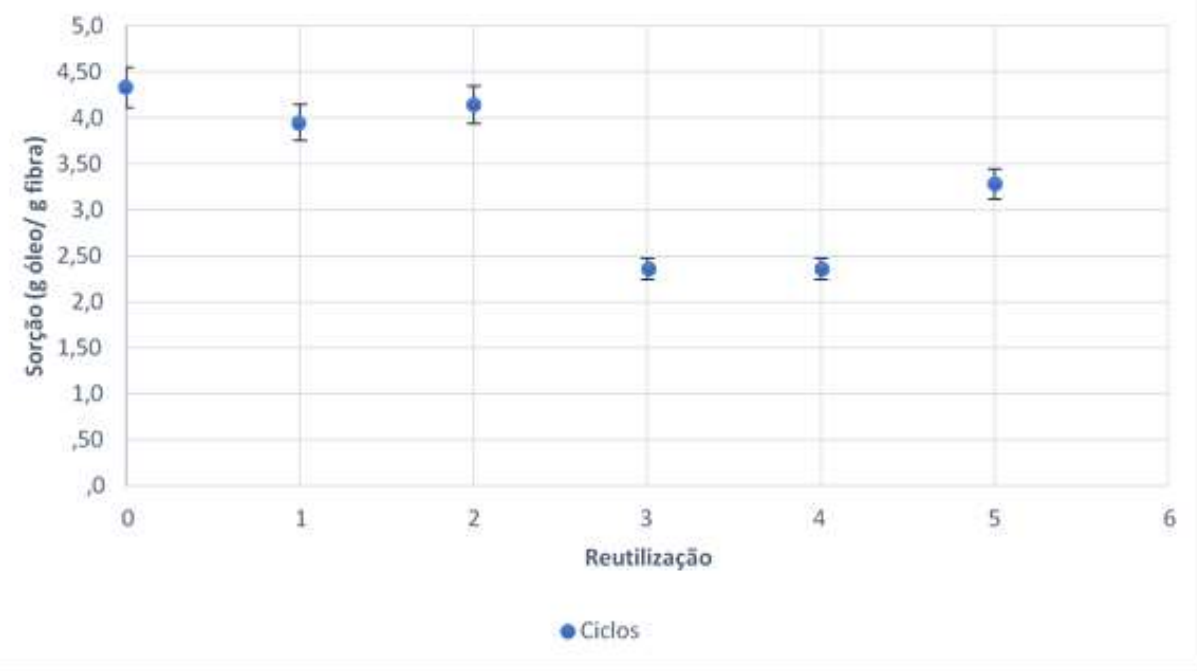

(b)

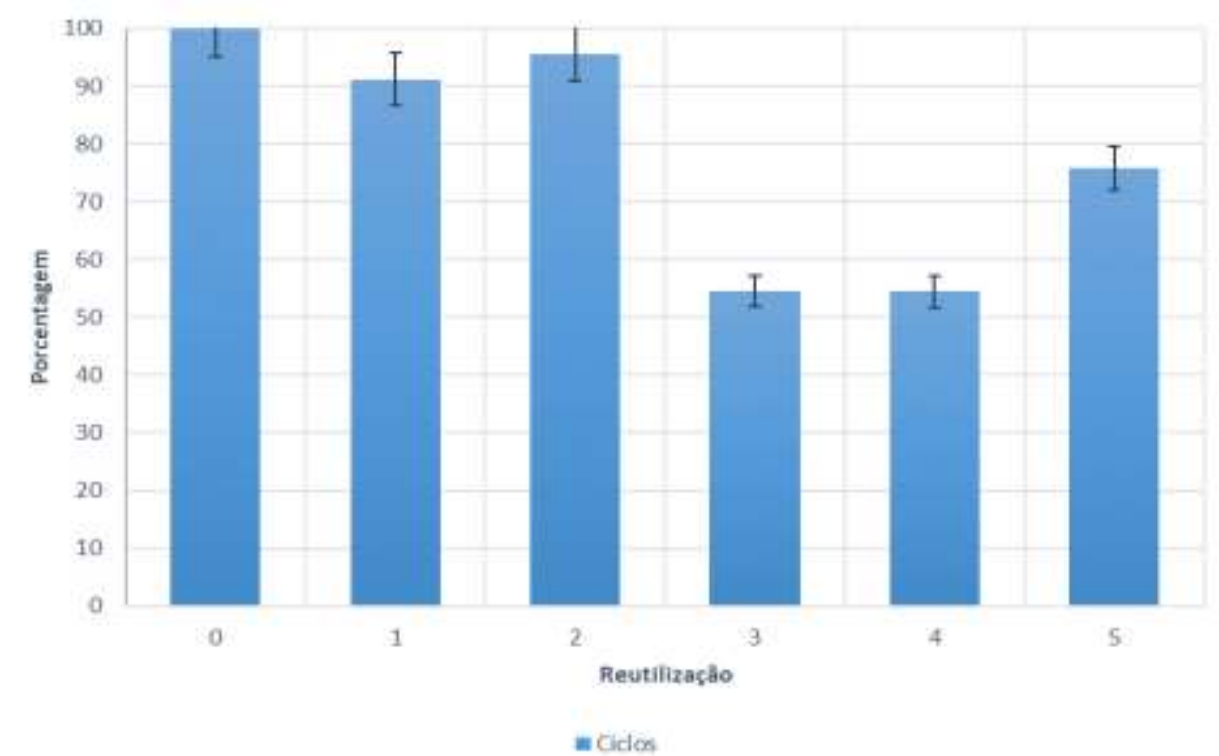

Fonte: Autores.

Tabela 4: Valores da sorção e da massa de óleo da Bacia de Campos pela fibra de sisal avaliados sob seis ciclos subsequentes.

\begin{tabular}{c|cccc}
\hline Ciclo & $\begin{array}{c}\text { Massa } \\
\text { de óleo (g) }\end{array}$ & $\begin{array}{c}\text { Sorção (g óleo/g fibra) } \\
\text { desidratada }\end{array}$ & $\begin{array}{c}\text { Desvio } \\
\text { padrão }\end{array}$ & $\begin{array}{c}\text { Porcentagem } \\
\text { de óleo adsorvida (\%) }\end{array}$ \\
\hline $\mathbf{1}$ & 2,165 & 4,330 & $\pm 0,002$ & 47,91 \\
\hline $\mathbf{2}$ & 1,974 & 3,947 & $\pm 0,003$ & 43,66 \\
\hline $\mathbf{3}$ & 2,071 & 4,141 & $\pm 0,002$ & 45,81 \\
\hline $\mathbf{4}$ & 1,180 & 2,360 & $\pm 0,002$ & 26,10 \\
\hline $\mathbf{5}$ & 1,178 & 2,357 & $\pm 0,003$ & 26,07 \\
\hline $\mathbf{6}$ & 1,640 & 3,280 & $\pm 0,003$ & 36,29 \\
\hline
\end{tabular}

Fonte: Autores.

Os resultados revelam o declínio de sorção do óleo da Bacia de Campos mais evidentes até a terceira reutilização (quarto ciclo). Nos três últimos ciclos, não houve uma variação acentuada, mantendo a redução próxima a constância. Um 
comportamento semelhante foi observado por outros autores que utilizam diferentes fibras para adsorver petróleo e derivados (Wang et al., 2012; Abdullah et al., 2010). Após o sexto ciclo de sorção/ dessorção, a eficiência do sorvente atingiu valores significativos, obtendo 3,28 gramas óleo para cada grama da fibra vegetal $( \pm 0,003)$. Este resultado indica que após a quinta reciclagem da fibra, a sorção decaiu cerca de $12 \%$ da primeira sorção.

O declínio do potencial de sorção do óleo pela fibra de sisal com o aumento do número de ciclos, pode ser justificada principalmente pela quantidade de óleo remanescente após sucessivas sorções. Este perfil, confere uma redução da área superficial porosa disponível na fibra proveniente do óleo residual retida na amostra após a centrifugação, interferindo na próxima sorção.

\section{Considerações Finais}

A busca do petróleo, embora vantajosa no setor econômico-industrial, pode gerar um crescente risco de derramamento marinho na sua exploração, transporte e armazenamento. Embora haja atualmente uma ampla gama de tecnologias aplicadas, o emprego de diversas técnicas, além de caras, pode acentuar o impacto causado pelo petróleo derramado.

A utilização de adsorventes lignocelulósicos, é um método promissor, barato, ecologicamente sustentável e eficaz na remediação de derramamentos em ambiente marinho. Este tipo de tecnologia utiliza fibras constituídas majoritariamente por lignina, hemicelulose e, em maior percentual, a celulose. Os materiais adsorventes têm a capacidade de adsorver o petróleo devido a sua porosidade, e estudos de equilíbrio e de adsorção devem ser realizados para verificação do método de adsorção, físico ou químico, de acordo com a capacidade de equilíbrio e a quantidade máxima de adsorção em um determinado período.

Diversas pesquisas foram realizadas para aumentar o percentual de adsorção pelas fibras, diminuindo as características hidrofílicas causadas pela celulose, através de métodos químicos, físicos e, com maior destaque, o tratamento biológico. A hidrolise enzimática é uma tecnologia já aplicada em diversos setores industriais e, desta maneira, a sua aplicabilidade poderia evitar custos operacionais de instalações, além de não gerar resíduos, sendo ecologicamente recomendável.

O estudo de caso com a fibra de sisal valida a boa eficiência da fibra de sisal in natura no processo de adsorção do petróleo da Bacia de Campos. Os resultados de sorção quanto ao tempo de contato, propõe a necessidade de um tempo mínimo de 5 minutos para aplicabilidade da técnica remediadora, configurando outra vantagem no uso do adsorvente. A reciclagem da fibra de sisal após cinco reutilizações, obteve resultados promissores, em virtude do declínio de apenas $12 \%$ da primeira sorção. Esses resultados sugerem a aplicabilidade da fibra como uma potencial ferramenta de remediação de áreas impactadas, após eventuais derramamentos offshore.

Estudos acerca de tratamentos para aumentar a capacidade de sorção pelo material com tratamentos devem ser realizados em testes de bancada para aplicabilidade em escala real. Além disso, sugere-se ainda um estudo com outros tipos de óleos de diferentes bacias, bem como o seu reaproveitamento após a sorção.

\section{Referências}

Abdullah, M. A., Rahmah, A. U., \& Man, Z. (2010). Physicochemical and sorption characteristics of Malaysian Ceiba pentandra (L.) Gaertn. as a natural oil sorbent. Journal of Hazardous Materials, 177(1-3), 683-691.

Al Zubaidi, I., Zaffar, U., Mustafa, N., Varughese, V., Rashid, A., Shahid, E. E., Ahmed, R., Alharmoudi, A., \& Gomes. (2015). Adsorption Study of BioDegradable Natural Sorbents for Remediation of Water from Crude Oil. International Conference on Environmental Science and Technology, 84, $138-142$.

Annunciado, T. R., Sydenstricker, T. H. D., \& Amico, S. C. (2005). Experimental investigation of various vegetable fibers as sorbent materials for oil spills. Marine Pollution Bulletin, 50(11), 1340-1346. https://doi.org/10.1016/j.marpolbul.2005.04.043

ANP. (2021). Produção de petróleo e gás teve recorde em 2020 e aumentou 52,71\% em relação a 2010. https://www.gov.br/anp/ptbr/canais_atendimento/imprensa/noticias-comunicados/producao-de-petroleo-e-gas-teve-recorde-em-2020-e-aumentou-52-71-em-relacao-a-2010 
Barbosa, A. D. P. (2011). Características estruturais e propriedades de compósitos poliméricos reforçados com fibras de buriti. Tese (Doutorado em Engenharia e Ciência dos Materiais), Universidade Estadual do Norte Fluminense, Campos dos Goytacazes-RJ.

Barragan, O. L. V. (2012). Caracterização Geoquímica de óleos da América. Dissertação (Mestrado em Geoquímica do Petróleo e Ambiental), Universidade Federal da Bahia, Instituto de Geociência IGEO/ UFBA, Salvador-BA.

Berger, A. H., \& Bhown, A. S. (2011). Comparing physisorption and chemisorption solid sorbents for use separating CO2 from flue gas using temperature swing adsorption. Energy Procedia, 4, 562-567.

Cardoso, C. K. M., Cardoso, R. da P. G., \& Moreira, Í. T. A. (2018). Avaliação de sorventes naturais para remediação de petróleo derramado em águas marinhas costeiras: o estado da arte e um estudo de caso aplicado. Seminário estudantil de produção acadêmica (SEPA) 16, $178-197$.

CETESB. (2021). Limpeza de ambientes costeiros. http://cetesb.sp.gov.br/emergencias-quimicas/tipos-de-acidentes/vazamentos-de-oleo/acoes-deresposta/limpeza-de-ambientes-costeiros/

Dong, T., Xu, G., \& Wang, F. (2015). Oil spill cleanup by structured natural sorbents made from cattail fibers. Industrial Crops \& Products, 76, 25-33.

EPA. (2021). Overview of the Spill Prevention, Control, and Countermeasure (SPCC) Regulation. https://www.epa.gov/oil-spills-prevention-andpreparedness-regulations/overview-spill-prevention-control-and

Ferreira, R. T. (2009). Sorção de petróleo por fibras vegetais. Dissertação (Mestrado em Engenharia Mecânica), Universidade Federal do Rio Grande do Norte, Natal-RN.

García-Garrido, V. J., Ramos, A., Mancho, A. M., Coca, J., \& Wiggins, S. (2016). A dynamical systems perspective for a real-time response to a marine oil spill. Marine Pollution Bulletin, 112(1-2), 201-210. https://doi.org/10.1016/j.marpolbul.2016.08.018

Grote, M. et al. (2018). The potential for dispersant use as a maritime oil spill response measure in German waters. Marine Pollution Bulletin, 129(2), 623632.

ITOPF. (2020). Oil Tanker Spill Statistics. http://www.itopf.com/knowledge-resources/data-statistics/statistics/

Li, P., Cai, Q., Lin, W., Chen, B., \& Zhang, B. (2016). Offshore oil spill response practices and emerging challenges. Marine Pollution Bulletin, $110(1), 6-27$.

Lim, T. T., \& Huang, X. (2007). Evaluation of kapok (Ceiba pentandra (L.) Gaertn.) as a natural hollow hydrophobic-oleophilic fibrous sorbent for oil spill cleanup. Chemosphere, 66(5), 955-963.

Lopes, C. F., Milanelli, J. C. C., \& Poffo, I. R. F. (2007). Ambientes Costeiros contaminados por óleo Procedimentos de limpeza- Manual de orientação. (CETESB (ed.)).

Martin, A. R., Martins, M. A., \& Silva, O. R. R. F. (2009). Caracterização Química e Estrutural de Fibra de Sisal da Variedade Agave sisalana. Polímeros: Ciência e Tecnologia, 19, 40-46.

Oliveira, O. M. C. de, Antônio, A. F., Cerqueira, J. R., Soares, S. A. R., Garcia, K. S., Filho, A. P., de L. da S. Rosa, M., Suzart, C. M., de L. Pinheiro, L., \& Moreira, I. T. A. (2020). Environmental disaster in the northeast coast of Brazil: Forensic geochemistry in the identification of the source of the oily material. Marine Pollution Bulletin, 160(July), 111597. https://doi.org/10.1016/j.marpolbul.2020.111597

PETROBRÁS. (2018). Pré-Sal novo. https://petrobras.com.br/pt/nossas-atividades/areas-de-atuacao/exploracao-e-producao-de-petroleo-e-gas/presal/\#: : text=Nossa produção diária de petróleo,barris por dia em 2018.

Podgorski, D. C. et al. (2012). Heavy petroleum composition. 5. compositional and structural continuum of petroleum revealed. Acs Publications Journal Stars, 27, 1268-1276.

Queiroz, M. S. M. (2011). Hidrocarbonetos policíclicos aromáticos em sedimentos de fundo do estuário do rio Potengi, região da grande Natal (RN): implicações ambientais. Dissertação (Mestrado em Pesquisa e Desenvolvimento em Ciência e Engenharia de Petróleo), Universidade Federal do Rio Grande do Norte, Natal-RN.

Segundo, P. de O. (2011). Avaliação da Viabilidade do Sisal como Sorvente de Óleo. Monografia (Graduação em Engenharia Química), Universidade Federal do Rio Grande do Norte, Natal-RN.

Sousa, C. S. Jr. (2009). Comportamento reológico de dispersões aquosas em óleo leve pesado, In Anais, $5^{\circ}$ Congresso Brasileiro de Pesquisa e Desenvolvimento em Petróleo e Gás (pp. 7), Fortaleza-CE, Universidade Federal do Ceará. Ceará: Edições UFC.

Speight, J. G., \& El-Gendy, N. S. (2017). Introduction to petroleum biotechnology: Petroleum Composition and Properties. In Gulf Professional Publishing (pp. 1-39).

Trigo, G. R., Zock, J. P., \& Montes, I. I. (2018). Health Effects of Exposure to Oil Spills. Archivos de Bronconeumología, 43, 628-635.

Wang, J., Zheng, Y., \& Wang, A. (2012). Effect of kapok fiber treated with various solvents on oil absorbency. Industrial Crops and Products, 40 (1), 178 184.

Wang, J., \& Guo, X. (2020). Adsorption kinetic models: Physical meanings, applications, and solving methods. Journal of Hazardous Materials, $390,122156$.

Wong, C., McGowan, T., Bajwa, S. G., \& Bajwa, D. S. (2016). Impact of fiber treatment on the oil absorption characteristics of plant fibers. BioResources, 11(3), 6452-6463. 